\title{
Association of adiposity with hemoglobin levels in patients with chronic kidney disease not on dialysis
}

\author{
Hirokazu Honda ${ }^{1} \cdot$ Kota Ono $^{2} \cdot$ Tadao Akizawa $^{3} \cdot$ Kosaku Nitta $^{4}$. \\ Akira Hishida $^{5}$
}

Received: 13 June 2017/ Accepted: 18 October 2017/Published online: 4 November 2017

(c) The Author(s) 2017. This article is an open access publication

\begin{abstract}
Background In the general population, adiposity influences erythropoiesis and iron metabolism. We aimed to assess the relationships between adiposity [estimated by body mass index (BMI) and abdominal circumference (AC)] and biomarkers of erythropoiesis in patients with chronic kidney disease (CKD) not on dialysis.

Methods A total of 2322 patients from the Chronic Kidney Disease Japan Cohort study were included. Patients were grouped according to BMI (low: $<18.5 \mathrm{~kg} / \mathrm{m}^{2}$, normal: $18.5-24.5 \mathrm{~kg} / \mathrm{m}^{2}$, and high: $\geq 25 \mathrm{~kg} / \mathrm{m}^{2}$ ) and AC categories (large: $\geq 90 \mathrm{~cm}$ for men and $\geq 80 \mathrm{~cm}$ for women; small: $<90 \mathrm{~cm}$ and $<80 \mathrm{~cm}$, respectively). Body composition and laboratory data were assessed at baseline, and at 1 and 2 years of follow-up.
\end{abstract}

Electronic supplementary material The online version of this article (https://doi.org/10.1007/s10157-017-1501-y) contains supplementary material, which is available to authorized users.

The original version of this article was revised due to a retrospective Open Access order.

Hirokazu Honda

hondah@med.showa-u.ac.jp

1 Division of Nephrology, Department of Medicine, Showa University Koto Toyosu Hospital, 5-1-38, Toyosu, Koto-ku, Tokyo 135-8577, Japan

2 Clinical Research and Medical Innovation Center, Hokkaido University Hospital, Sapporo, Japan

3 Division of Nephrology, Department of Medicine, Showa University School of Medicine, Tokyo, Japan

4 Fourth Department of Internal Medicine, Tokyo Women's Medical University, Tokyo, Japan

5 Yaizu City Hospital, Yaizu, Japan
Results Multivariate regression analysis of the 3 timepoints showed that high BMI and large AC in male patients were significantly associated with higher hemoglobin levels. Hemoglobin levels were lower in female patients with low BMI and small AC than that in female patients with normal BMI and large AC, respectively; however, hemoglobin levels plateaued above a threshold of $25 \mathrm{~kg} / \mathrm{m}^{2}$ for BMI and $80 \mathrm{~cm}$ for AC. While BMI and AC were positively associated with C-reactive protein levels, they were not associated with levels of transferrin saturation, ferritin, and erythropoietin in multivariate models.

Conclusions Body composition appears to be associated with erythropoiesis; however, adiposity may be only associated with increased erythropoiesis in male patients. In addition, body composition does not appear to hamper iron metabolism in CKD patients not on dialysis.

Keywords Abdominal circumference - Body mass index . Erythropoiesis · Inflammation · Nutrition

\section{Introduction}

Anemia is a major complication in patients with advanced chronic kidney disease (CKD) and an independent risk for cardiovascular mortality and end-stage kidney disease [1-3]. While inadequate erythropoietin (EPO) production, or EPO deficiency, in a uremic condition is a main cause of anemia in CKD, a multitude of factors such as malnutrition, chronic inflammation, and a low absorption of nutrients (including iron, folic acid, and vitamin B12) influence erythropoiesis in these patients [4].

Erythropoiesis is more common in overweight and obese individuals than in individuals with normal body weight [5-7]. This phenomenon is thought to be caused by abdominal 
fat. Abdominal fat is a source of adipokines, such as leptin and adiponectin, and is a cause of diminished insulin response; these factors can lead to erythropoiesis [8-14]. Adiposity is also associated with iron metabolism [15-18]. As abdominal fat is a source of inflammatory cytokines, adiposity causes a state of chronic, low-grade inflammation, which results in the over-production of hepcidin-25 [7]. Iron metabolism is regulated by hepcidin-25; a high level of hepcidin-25 can lead to decreased serum iron levels, increased reticuloendothelial iron accumulation, and/or decreased intestinal iron absorption $[19,20]$. Consequently, overweight and obese individuals may develop abnormal iron metabolism. However, the prevalence of anemia in overweight and obese individuals is not higher than that in individuals with normal body weight. Although individuals who are overweight or obese show high levels of iron deficiency, their red blood cell (RBC) count and hemoglobin levels are not always in the anemic range [15, 17].

Thus, adiposity is possibly associated with erythropoiesis and iron deficiency in patients with CKD. However, insulin and adipokine metabolism in CKD patients do not resemble that in the non-CKD population [21-23]. Moreover, patients with advanced $\mathrm{CKD}$ often show inadequate EPO production and absolute or functional iron deficiency. Levels of hepcidin-25 increase with increasing CKD severity [24, 25], and patients with advanced CKD may show chronic anemia due to the over-production of hepcidin-25 caused by chronic inflammation [24].

Based on these findings and issues, we hypothesized that adiposity would be associated with erythropoiesis and/or impaired iron metabolism in patients with CKD, and that these associations would be influenced by CKD severity. Therefore, we evaluated the associations between adiposity (estimated by body mass index [BMI] and abdominal circumference $[\mathrm{AC}]$ ) and biomarkers of erythropoiesis in patients with CKD who were not on dialysis.

\section{Methods}

\section{Study design}

This study was performed as part of the CKD-JAC study, which has been previously described [26, 27]. Briefly, the CKD-JAC study was designed as a multicenter prospective cohort study; 3087 participants who were Japanese patients with CKD not on dialysis were included in the original study [28, 29]. The inclusion criteria were age between 20 and 75 years and low estimated glomerular filtration rate (eGFR) (10-59 $\mathrm{mL} / \mathrm{min} / 1.73 \mathrm{~m}^{2}$ ), which was calculated using the following formula: eGFR $\left(\mathrm{mL} / \mathrm{min} / 1.73 \mathrm{~m}^{2}\right)=194$ age $^{-0.287}$ serum creatinine ${ }^{-1.094}(\times 0.739$ for women $)$.

Patients were excluded if they had polycystic kidney disease, human immunodeficiency virus infection, cirrhosis, or active cancer; had undergone cancer treatment within the past 2 years or chronic dialysis; received a transplant; or were pregnant. The protocol was approved by the ethics committee of each participating institution, and the study was conducted in accordance with the Declaration of Helsinki. All patients provided written informed consent. Registration began in September 2007, and 3087 patients were recruited. However, several patients dropped out or were excluded due to exclusion criteria $(n=27)$, unavailable baseline data $(n=25)$, withdrawn consent $(n=59)$, a lack of hospital visits during the observation period $(n=4)$, or death during the study period $(n=1)$. In addition, 5 patients were excluded on the instruction of the attending physician. Therefore, 2966 patients were finally enrolled in the CKD-JAC study [28, 29].

As the present study aimed to assess the impact of adiposity on biomarkers of erythropoiesis and iron metabolism, patients who were treated with an erythropoietin stimulating agent (ESA) were also excluded from analysis. Thus, the BMI cohort comprised 2322 patients with height and weight measurements, and the AC cohort comprised 1625 patients with AC measurements. The WHO international criteria for BMI were used to estimate body composition [30], and the BMI cohort was grouped into 3 categories: low BMI $\left(<18.5 \mathrm{~kg} / \mathrm{m}^{2}\right)$, normal BMI $\left(18.5-25 \mathrm{~kg} / \mathrm{m}^{2}\right)$, and high BMI $\left(\geq 25 \mathrm{~kg} / \mathrm{m}^{2}\right)$. The AC cohort was grouped into 2 categories using the WHOrecommended cut-off points for the estimation of abdominal obesity in Asian populations [31]: small AC $(<90 \mathrm{~cm}$ for men and $<80 \mathrm{~cm}$ for women) and large $\mathrm{AC}(\geq 90 \mathrm{~cm}$ for men and $\geq 80 \mathrm{~cm}$ for women). AC was measured around the waist, midway between the top of the hip bone and the bottom of the ribs.

\section{Covariates}

Patient characteristics; comorbidities, such as diabetes mellitus (DM) and history of cardiovascular disease; medication use; and routine laboratory data were extracted from the electronic health records at baseline, and at 1 and 2 years of follow-up. Stored frozen serum samples were used to measure intact fibroblast growth factor (intactFGF23) at the 3 time-points, intact parathyroid hormone and 25-hydroxyvitamin D at baseline, and EPO concentration at 1 year. Intact-FGF23 was measured using a chemiluminescent enzyme immunoassay (Kyowa Medex, Tokyo, Japan), 25-hydroxyvitamin D was measured using a chemiluminescent immunoassay (Liaison 25-hydroxyvitamin D Total assay, DiaSorin, Italy), and EPO concentration was measured using a sandwich radio immunoassay, performed at LSI Medicine Corporation (Tokyo, Japan). 


\section{Statistics}

Data are presented as mean \pm standard deviation (SD) or medians (range), unless otherwise noted. $p<0.05$ was considered indicative of statistical significance. Group differences were evaluated using analyses of variance for normally distributed variables, the Kruskal-Wallis test for non-normally distributed variables, and the $\chi^{2}$ test for nominal variables. Independent associations between body composition measures and levels of hemoglobin and iron metabolism biomarkers were assessed at baseline, 1 year, and 2 years using multiple linear regression models. In addition, associations body composition measures and levels of hemoglobin, iron metabolism biomarkers, and CRP were assessed using multivariate restricted cubic curves with 4 knots located at the 5th, 35th, 65th and 95th percentiles of BMI or AC. Tests for nonlinearity were performed by comparing a model with a linear term to a model with linear and restricted cubic spline terms. When the test for nonlinearity was not significant, a test for linearity was performed by comparing a model with the linear term to a model without the linear term. Associations between BMI and AC categories and hemoglobin levels were estimated using multivariate repeated-measures analyses. Longitudinal analyses were performed using mixed-effect models with baseline covariates and timepoints; the intercept and time-points were also treated as random effects. Interactions between baseline covariates and time-points were considered to reflect a longitudinal effect. All data were analyzed using SAS software (version 9.4, SAS Institute Inc., Cary, North Carolina, USA).

\section{Results}

\section{Patient characteristics and laboratory findings}

A total of 2,322 patients from the Chronic Kidney Disease Japan Cohort (CKD-JAC) study [26, 27] with height and weight measurements were selected as the BMI cohort, and 1,625 patients with AC measurements were selected as the AC cohort. Patient characteristics and laboratory data at baseline are shown according to BMI and AC in Tables 1 and S1, respectively. Patients with high BMI showed increased RBC count and increased levels of hemoglobin, ferritin, and C-reactive protein (CRP) (Tables 1 and 2). In both sexes, the characteristics of patients with a large AC resembled those of patients with a high BMI (Supplementary Tables S1-1 and S1-2). Ferritin levels in patients with a high BMI were higher than those in other BMI categories (Tables 1 and 2). Ferritin levels in female patients, but not male patients, with a large AC were higher than those in patients with a small AC (Supplementary Tables S1-1 and S1-2).

\section{Associations between body composition measures and hemoglobin level}

Independent associations between body composition and hemoglobin level were evaluated at baseline, and at 1 and 2 years of follow-up in multiple linear regression models, adjusting for various potential cofounders. In addition, multivariate restricted cubic curves were constructed. Hemoglobin levels were significantly increased in male patients with high BMI compared to those in male patients with normal BMI (Table 3), with hemoglobin level increasing linearly with increasing BMI (Fig. 1). Hemoglobin levels were reduced in female patients with low BMI compared to those in female patients with normal BMI (Table 3). Although the association between BMI and hemoglobin level was linear at lower BMI levels in female patients, the spline curve tended to plateau once a BMI threshold of $25 \mathrm{~kg} / \mathrm{m}^{2}$ was reached (Fig. 1).

The associations between $\mathrm{AC}$ and hemoglobin level resembled those for BMI and hemoglobin level (Supplementary Table S2). Hemoglobin levels were significantly increased in male patients with large AC compared to those in male patients with small AC (Supplementary Table S2). Although hemoglobin levels increased linearly with increasing AC in both sexes (Supplementary Figure S1), hemoglobin levels plateaued once an AC threshold of $80 \mathrm{~cm}$ was reached in female patients (Supplementary Figure S1).

Given the reduced number of patients in more complicated models, the patient characteristics and laboratory data were compared between those included and excluded from Model 2. The results are provided in Supplementary Tables S3-1 and S3-2.

Multivariate repeated-measures analyses were performed to evaluate cross-sectional and longitudinal effects in the associations between baseline body composition and hemoglobin levels at 3 time-points (baseline, 1 year of follow-up, and 2 years of follow-up). Male patients with low BMI, male patients with high BMI, and male patients with large $\mathrm{AC}$ showed independent associations with baseline hemoglobin level as cross-sectional effects (Supplementary Tables S4 and S5). These associations did not change 1 and 2 years later, as indicated in the analysis of longitudinal effects (Supplementary Tables S4 and S5).

\section{Associations between body composition measures and hemoglobin levels according to CKD stage}

Spline curves for the association between BMI and hemoglobin level were linear in patients with stages 3 and 
Table 1 Male patient characteristics and laboratory findings according to body mass index

\begin{tabular}{|c|c|c|c|c|c|}
\hline & All $(n=1497)$ & Low BMI $(n=53)$ & $\begin{array}{l}\text { Normal BMI } \\
(n=914)\end{array}$ & $\begin{array}{l}\text { High BMI } \\
(n=530)\end{array}$ & $p^{*}$ \\
\hline Age (years) & $60.9 \pm 11.2$ & $58.6 \pm 14.9$ & $61.8 \pm 10.8$ & $59.6 \pm 11.3$ & $<0.001$ \\
\hline Diabetes mellitus $(n, \%)$ & $603(40.3)$ & $20(27.7)$ & $323(35.3)$ & $260(49.1)$ & $<0.001$ \\
\hline Height $(\mathrm{cm})$ & $166.4 \pm 6.4$ & $166.2 \pm 7.4$ & $166.2 \pm 6.3$ & $166.7 \pm 6.6$ & 0.396 \\
\hline Weight (kg) & $66.7 \pm 11.4$ & $48.2 \pm 4.6$ & $61.6 \pm 6.5$ & $77.4 \pm 9.9$ & $<0.001$ \\
\hline Body mass index $\left(\mathrm{kg} / \mathrm{m}^{2}\right)$ & $23.73(21.67-26.04)$ & $17.45(16.98-18.07)$ & $\begin{array}{l}22.37 \\
\quad(21.01-23.69)\end{array}$ & $27.12(25.82-28.91)$ & $<0.001$ \\
\hline Abdominal circumference $(\mathrm{cm})$ & $85.5 \pm 9.6[1061]$ & $70.7 \pm 4.2$ & $82.6 \pm 6.2$ & $95.2 \pm 8.3$ & $<0.001$ \\
\hline Cause of CKD $(n, \%)$ & & & & & $<0.001$ \\
\hline CGN & $570(38.1)$ & $21(39.6)$ & $385(42.1)$ & $164(30.9)$ & \\
\hline DMN & $323(21.6)$ & $10(18.9)$ & $162(17.7)$ & $151(28.5)$ & \\
\hline Nephrosclerosis & $350(23.4)$ & $12(22.6)$ & $194(21.2)$ & $144(27.2)$ & \\
\hline Other diseases & $254(17.0)$ & $10(18.9)$ & $173(18.9)$ & $71(13.4)$ & \\
\hline CKD stage $(n, \%)$ & & & & & 0.282 \\
\hline $3 \mathrm{~A}$ & 174 (11.6) & $2(3.8)$ & $102(11.2)$ & $70(13.2)$ & \\
\hline 3B & $592(39.5)$ & $25(47.2)$ & $350(38.3)$ & $217(40.9)$ & \\
\hline 4 & $560(37.4)$ & $19(35.8)$ & $352(38.5)$ & $189(35.7)$ & \\
\hline 5 & $171(11.4)$ & $7(13.2)$ & $110(12.0)$ & $54(10.2)$ & \\
\hline History of CVD (yes, \%) & $394(26.3)$ & $13(24.5)$ & $233(25.5)$ & $148(27.9)$ & 0.573 \\
\hline ACE inhibitor/ARB (yes, \%) & $1252(83.6)$ & $37(69.8)$ & $750(82.1)$ & $465(87.7)$ & $<0.001$ \\
\hline Ferrotherapy $(n, \%)$ & $48(3.2)$ & $2(3.8)$ & $31(3.4)$ & $15(2.8)$ & $<0.001$ \\
\hline Red blood cell count $\left(10^{4} / \mu \mathrm{L}\right)$ & $404.8 \pm 60.6[1474]$ & $387.9 \pm 63.4$ & $396.9 \pm 58.4$ & $420.1 \pm 61.1$ & $<0.001$ \\
\hline Hemoglobin (g/dL) & $12.72 \pm 1.84[1474]$ & $12.16 \pm 1.84$ & $12.48 \pm 1.76$ & $13.20 \pm 1.82$ & $<0.001$ \\
\hline Serum albumin (g/dL) & $3.95 \pm 0.43[1450]$ & $3.94 \pm 0.44$ & $3.97 \pm 0.44$ & $3.96 \pm 0.42$ & 0.788 \\
\hline Serum creatinine $(\mathrm{mg} / \mathrm{dL})$ & $2.20 \pm 1.01$ & $2.33 \pm 1.02$ & $2.20 \pm 0.99$ & $2.18 \pm 1.03$ & 0.554 \\
\hline eGFR $\left(\mathrm{ml} / \mathrm{min} / 1.73 \mathrm{~m}^{2}\right)$ & $30.44 \pm 11.87$ & $28.57 \pm 10.52$ & $30.11 \pm 11.69$ & $31.19 \pm 12.27$ & 0.129 \\
\hline Serum cystatin $\mathrm{C}(\mathrm{mg} / \mathrm{L})$ & $1.807 \pm 0.652[1397]$ & $1.964 \pm 0.603$ & $1.824 \pm 0.664$ & $1.764 \pm 0.633$ & 0.071 \\
\hline Serum corrected calcium $(\mathrm{mg} / \mathrm{dL})$ & $9.17 \pm 0.43[1341]$ & $9.14 \pm 0.38$ & $9.17 \pm 0.42$ & $9.17 \pm 0.45$ & 0.843 \\
\hline Serum phosphate (mg/dL) & $3.54 \pm 0.70[1313]$ & $3.77 \pm 0.81$ & $3.52 \pm 0.67$ & $3.52 \pm 0.73$ & $<0.001$ \\
\hline Intact parathyroid hormone (pg/mL) & $74.0(50.0-114.0)[1397]$ & $101.0(61.0-140.0)$ & $74.0(50.0-112.0)$ & $73.0(50.0-111.0)$ & $0.056^{\mathrm{a}}$ \\
\hline 25-Hydroxyvitamin D (ng/mL) & $\begin{array}{l}16.20(10.00-24.05) \\
{[1364]}\end{array}$ & $14.35(9.30-20.20)$ & $\begin{array}{l}17.10 \\
(10.30-25.10)\end{array}$ & $15.10(9.70-22.60)$ & $0.012^{\mathrm{a}}$ \\
\hline Fibroblast growth factor $23(\mathrm{pg} / \mathrm{mL})$ & $56.6(40.5-86.3)[1375]$ & $54.3(43.2-98.8)$ & $56.6(39.9-83.5)$ & $56.7(41.4-91.5)$ & $0.505^{\mathrm{a}}$ \\
\hline Serum iron $(\mu \mathrm{g} / \mathrm{dL})$ & $87.7 \pm 30.8$ [795] & $86.1 \pm 27.5$ & $87.1 \pm 31.2$ & $88.8 \pm 30.4$ & 0.745 \\
\hline Total iron-binding capacity $(\mu \mathrm{g} / \mathrm{dL})$ & $291.9 \pm 49.9[526]$ & $295.3 \pm 91.8$ & $287.5 \pm 48.6$ & $299.6 \pm 46.4$ & 0.030 \\
\hline Transferrin saturation (\%) & $31.23 \pm 11.73[525]$ & $29.98 \pm 11.56$ & $31.60 \pm 12.25$ & $30.68 \pm 10.78$ & 0.634 \\
\hline Serum ferritin $(\mathrm{ng} / \mathrm{mL})$ & $\begin{array}{l}116.50(64.00-194.45) \\
{[756]}\end{array}$ & $\begin{array}{l}93.00 \\
\quad(40.00-148.20)\end{array}$ & $\begin{array}{l}113.00 \\
\quad(61.00-187.00)\end{array}$ & $\begin{array}{l}125.50 \\
(74.00-208.00)\end{array}$ & $0.021^{\mathrm{a}}$ \\
\hline C-reactive protein $(\mathrm{mg} / \mathrm{dL})$ & $\begin{array}{l}0.100(0.040-0.200) \\
\quad[1139]\end{array}$ & $0.080(0.030-0.200)$ & $\begin{array}{l}0.090 \\
(0.040-0.200)\end{array}$ & $0.120(0.050-0.230)$ & $<0.001^{\mathrm{a}}$ \\
\hline $\begin{array}{l}\text { Urine albumin-to-creatinine ratio } \\
(\mathrm{mg} / \mathrm{g} \mathrm{Cr})\end{array}$ & $\begin{array}{l}508.80(108.20-1367.60) \\
\quad[1369]\end{array}$ & $\begin{array}{l}671.15 \\
\quad(163.90-1348.20)\end{array}$ & $\begin{array}{l}433.55 \\
\quad(89.10-1184.20)\end{array}$ & $\begin{array}{l}619.30 \\
\quad(139.00-1661.60)\end{array}$ & 0.001 \\
\hline
\end{tabular}

Values are expressed as $n(\%)$, mean $\pm \mathrm{SD}$, or median (interquartile range). * $p$ value for BMI-group differences. The number of participants with non-missing data is shown in []; proportions are based on non-missing data

$B M I$ body mass index, low $B M I<18.5 \mathrm{~kg} / \mathrm{m}^{2}$, normal $B M I 18.5-24.5 \mathrm{~kg} / \mathrm{m}^{2}$, high $B M I \geq 25 \mathrm{~kg} / \mathrm{m}^{2}, C K D$ chronic kidney disease, $C G N$ chronic glomerulonephritis, $D M N$ diabetic nephropathy, $C V D$ cardiovascular disease, $A C E$ inhibitor angiotensin-converting enzyme inhibitor, $A R B$ angiotensin II receptor blocker, eGFR estimated glomerular filtration rate

${ }^{a} p$ values were calculated using the Kruskal-Wallis test 
Table 2 Female patient characteristics and laboratory findings according to body mass index

\begin{tabular}{|c|c|c|c|c|c|}
\hline & All $(n=825)$ & Low BMI $(n=96)$ & $\begin{array}{l}\text { Normal BMI } \\
(n=502)\end{array}$ & High BMI $(n=227)$ & $p^{*}$ \\
\hline Age (years) & $58.2 \pm 12.2$ & $54.4 \pm 14.2$ & $58.5 \pm 11.9$ & $59.3 \pm 11.7$ & 0.004 \\
\hline Diabetes mellitus $(n, \%)$ & $238(28.8)$ & $22(22.9)$ & $112(22.3)$ & $104(45.8)$ & $<0.001$ \\
\hline Height $(\mathrm{cm})$ & $153.8 \pm 6.0$ & $155.2 \pm 6.4$ & $153.9 \pm 6.0$ & $153.1 \pm 5.7$ & 0.019 \\
\hline Weight (kg) & $54.3 \pm 10.4$ & $40.6 \pm 5.3$ & $51.5 \pm 5.6$ & $66.5 \pm 8.2$ & $<0.001$ \\
\hline Body mass index $\left(\mathrm{kg} / \mathrm{m}^{2}\right)$ & $22.54(19.98-25.44)$ & $\begin{array}{l}17.43 \\
\quad(16.12-18.01)\end{array}$ & $\begin{array}{l}21.77 \\
\quad(20.13-23.23)\end{array}$ & $27.69(26.22-29.61)$ & $<0.001$ \\
\hline Abdominal circumference $(\mathrm{cm})$ & $80.1 \pm 11.8[553]$ & $66.5 \pm 6.5$ & $77.3 \pm 8.4$ & $92.4 \pm 9.0$ & $<0.001$ \\
\hline Cause of CKD $(n, \%)$ & & & & & $<0.001$ \\
\hline CGN & $470(57.0)$ & $52(54.2)$ & $307(61.2)$ & $111(48.9)$ & \\
\hline DMN & $109(13.2)$ & $6(6.3)$ & $48(9.6)$ & $55(24.2)$ & \\
\hline Nephrosclerosis & $94(11.4)$ & $9(9.4)$ & $52(10.4)$ & $33(14.5)$ & \\
\hline Other diseases & $152(18.4)$ & $29(30.2)$ & $95(18.9)$ & $28(12.3)$ & \\
\hline CKD stage $(n, \%)$ & & & & & 0.003 \\
\hline $3 \mathrm{~A}$ & $104(12.6)$ & $5(5.2)$ & 58 (11.6) & $41(18.1)$ & \\
\hline 3B & $306(37.1)$ & $28(29.2)$ & $198(39.4)$ & $80(35.2)$ & \\
\hline 4 & $322(39.0)$ & $46(47.9)$ & $197(39.2)$ & $79(34.8)$ & \\
\hline 5 & $93(11.3)$ & $17(17.7)$ & $49(9.8)$ & $27(11.9)$ & \\
\hline History of CVD (yes, \%) & $644(78.1)$ & $8(8.3)$ & $70(13.9)$ & $35(15.4)$ & 0.231 \\
\hline ACE inhibitor/ARB (yes, \%) & $200(24.2)$ & $53(59.4)$ & $386(76.9)$ & $201(88.5)$ & $<0.001$ \\
\hline Ferrotherapy $(n, \%)$ & $83(10.1)$ & $16(16.7)$ & $47(9.4)$ & $20(8.8)$ & 0.071 \\
\hline Red blood cell count $\left(10^{4} / \mu \mathrm{L}\right)$ & $382.9 \pm 53.9[809]$ & $360.3 \pm 49.3$ & $382.8 \pm 54.7$ & $392.7 \pm 51.1$ & $<0.001$ \\
\hline Hemoglobin (g/dL) & $11.64 \pm 1.45[809]$ & $11.08 \pm 1.33$ & $11.62 \pm 1.43$ & $11.94 \pm 1.50$ & $<0.001$ \\
\hline Serum albumin $(\mathrm{g} / \mathrm{dL})$ & $4.00 \pm 0.39[800]$ & $4.02 \pm 0.37$ & $4.02 \pm 0.38$ & $3.93 \pm 0.39$ & 0.011 \\
\hline Serum creatinine $(\mathrm{mg} / \mathrm{dL})$ & $1.69 \pm 0.77$ & $1.96 \pm 0.86$ & $1.65 \pm 0.73$ & $1.66 \pm 0.81$ & 0.001 \\
\hline eGFR $\left(\mathrm{ml} / \mathrm{min} / 1.73 \mathrm{~m}^{2}\right)$ & $30.53 \pm 12.20$ & $26.51 \pm 10.95$ & $30.86 \pm 12.00$ & $31.50 \pm 12.85$ & 0.002 \\
\hline Serum cystatin $\mathrm{C}(\mathrm{mg} / \mathrm{L})$ & $1.712 \pm 0.620[772]$ & $1.926 \pm 0.628$ & $1.673 \pm 0.612$ & $1.708 \pm 0.618$ & 0.002 \\
\hline Serum corrected calcium (mg/dL) & $9.28 \pm 0.455[725]$ & $9.22 \pm 0.53$ & $9.28 \pm 0.43$ & $9.31 \pm 0.46$ & 0.348 \\
\hline Serum phosphate $(\mathrm{mg} / \mathrm{dL})$ & $3.73 \pm 0.60[715]$ & $3.83 \pm 0.71$ & $3.71 \pm 0.59$ & $3.73 \pm 0.57$ & 0.258 \\
\hline Intact parathyroid hormone $(\mathrm{pg} / \mathrm{mL})$ & $77.0(54.0-122.0)[773]$ & $102.0(59.0-160.0)$ & $76.0(53.0-111.0)$ & $77.0(56.0-125.5)$ & 0.024 \\
\hline 25-Hydroxyvitamin D (ng/mL) & $13.45(8.80-19.00)[754]$ & $12.85(8.05-18.75)$ & $13.70(9.40-19.40)$ & $12.90(8.20-17.90)$ & $<0.012^{2}$ \\
\hline Fibroblast growth factor $23(\mathrm{pg} / \mathrm{mL})$ & $49.7(36.6-73.8)[762]$ & $49.6(36.4-69.8)$ & $48.4(36.2-73.4)$ & $52.9(38.4-79.5)$ & $0.266^{\circ}$ \\
\hline Serum iron $(\mu \mathrm{g} / \mathrm{dL})$ & $79.1 \pm 32.5[446]$ & $79.8 \pm 30.4$ & $78.4 \pm 30.9$ & $80.2 \pm 36.8$ & 0.867 \\
\hline Total iron-binding capacity $(\mu \mathrm{g} / \mathrm{dL})$ & $301.7 \pm 54.4[286]$ & $305.4 \pm 69.4$ & $300.6 \pm 54.5$ & $302.6 \pm 45.8$ & 0.877 \\
\hline Transferrin saturation $(\%)$ & $28.00 \pm 12.25[285]$ & $28.77 \pm 13.60$ & $27.35 \pm 11.58$ & $29.14 \pm 13.12$ & 0.532 \\
\hline Serum ferritin $(\mathrm{ng} / \mathrm{mL})$ & $\begin{array}{l}60.65(32.85-120.30) \\
{[412]}\end{array}$ & $\begin{array}{l}43.50 \\
(18.45-117.55)\end{array}$ & $\begin{array}{l}57.64 \\
\quad(31.50-115.50)\end{array}$ & $\begin{array}{l}76.45 \\
\quad(41.90-126.20)\end{array}$ & $0.016^{\circ}$ \\
\hline C-reactive protein $(\mathrm{mg} / \mathrm{dL})$ & $\begin{array}{l}0.070(0.030-0.200) \\
\quad[611]\end{array}$ & $\begin{array}{l}0.040 \\
\quad(0.020-0.115)\end{array}$ & $\begin{array}{l}0.060 \\
\quad(0.030-0.150)\end{array}$ & $0.110(0.050-0.280)$ & $<0.001^{\circ}$ \\
\hline $\begin{array}{l}\text { Urine albumin-to-creatinine ratio } \\
(\mathrm{mg} / \mathrm{g} \mathrm{Cr})\end{array}$ & $\begin{array}{l}430.20(111.10-1087.50) \\
\text { [761] }\end{array}$ & $\begin{array}{l}406.40 \\
\quad(147.25-817.80)\end{array}$ & $\begin{array}{l}383.45 \\
\quad(95.20-1032.60)\end{array}$ & $\begin{array}{l}585.20 \\
\quad(147.10-1495.10)\end{array}$ & 0.008 \\
\hline
\end{tabular}

Values are expressed as $\mathrm{n}(\%)$, mean $\pm \mathrm{SD}$, or median (inter quartile range). *P value for BMI-group differences. The number of participants with non-missing data is shown in []; proportions are based on non-missing data. ${ }^{\text {a }} \mathrm{P}$ values were calculated using the Kruskal-Wallis test. BMI: body mass index, low BMI: $<18.5 \mathrm{~kg} / \mathrm{m}^{2}$, normal BMI: $18.5-24.5 \mathrm{~kg} / \mathrm{m}^{2}$, high BMI: $\geq 25 \mathrm{~kg} / \mathrm{m}^{2}$, CKD: chronic kidney disease, CGN: chronic glomerulonephritis, DMN: diabetic nephropathy, CVD: cardiovascular disease, ACE inhibitor: angiotensin-converting enzyme inhibitor, ARB: angiotensin II receptor blocker, eGFR: estimated glomerular filtration rate

$4 \mathrm{CKD}$, but not in patients with stage 5 CKD (Supplementary Figure S2). In addition, the spline curves for the association between hemoglobin levels and AC according to CKD stage resembled those for BMI (Supplementary Figure S3). 


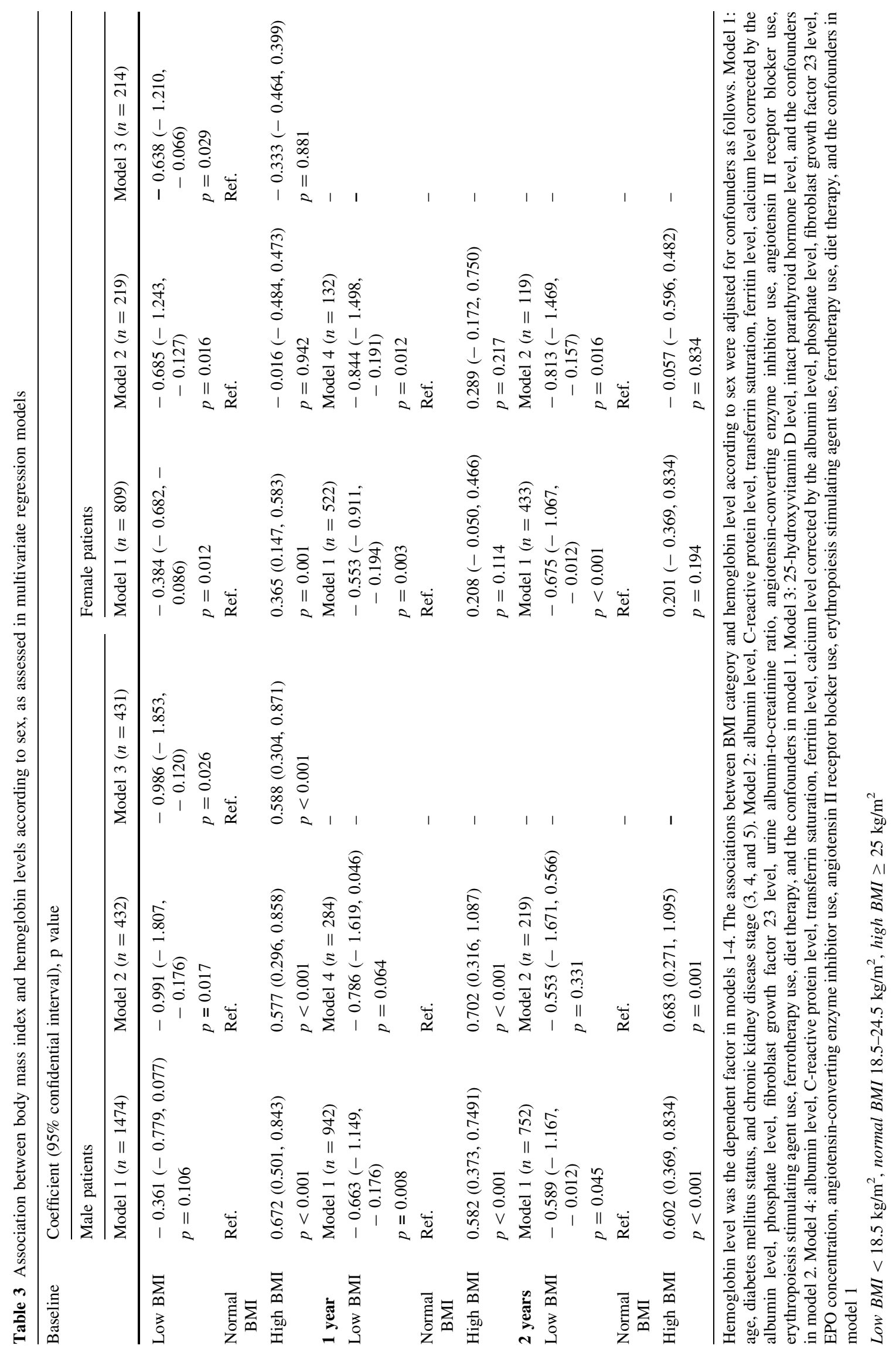




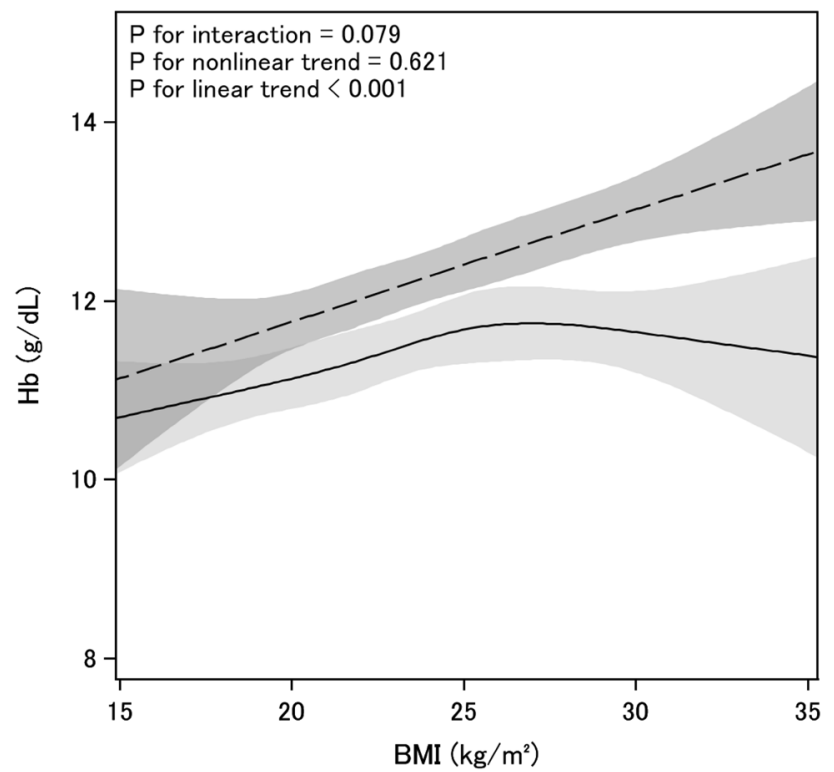

Fig. 1 Spline curves show the associations between body mass index (BMI) and hemoglobin ( $\mathrm{Hb}$ ) level at baseline, according to sex [solid lines, female patients $(n=219)$; dashed lines, male patients $(n=435)]$. Spline curves are adjusted for age (in 10-year increments); diabetes mellitus status; chronic kidney disease stage (3, 4, and 5); levels of albumin, $\log$ C-reactive protein, $\log$ fibroblast growth factor 23, log ferritin, transferrin saturation, albumin-adjusted calcium, and phosphate; medication use (angiotensin-converting enzyme inhibitor inhibitors and angiotensin II receptor blockers); and ferrotherapy use

\section{Association between body composition and erythropoietin concentration at 1 year of follow- up}

EPO concentrations at 1 year of follow-up according to body composition and sex are provided in Supplementary Table S6. In the multivariate regression models, there were no significant associations between EPO levels and either BMI or AC (Supplementary Table S7, models 2 and 4; Supplementary Fig. 4). However, linear associations between EPO level and both BMI and AC were confirmed in patients with stage $3 \mathrm{CKD}$; these associations disappeared in patients with stages 4 and 5 CKD (Supplementary Figure S5).

\section{Association between body composition and levels of transferrin saturation, ferritin, and C-reactive protein}

In multiple linear regression analyses, AC was not associated with transferrin saturation and ferritin levels at baseline, and at 1 and 2 years of follow-up (Supplementary Tables S8 and S9). In contrast, BMI was associated with ferritin levels (Tables 1 and 2). Spline curves showing the associations between body composition measures and transferrin saturation and ferritin levels, are shown in Supplementary Figures S6 (BMI) and S7 (AC). Logtransformed CRP levels increased linearly with increasing $\mathrm{BMI}$ and $\mathrm{AC}$ at baseline in patients at all stages of CKD (Supplementary Figure S8).

\section{Discussion}

To our knowledge, the present study is the first to analyze the association between body composition and biomarkers of erythropoiesis in patients with CKD who are not on dialysis. The present results demonstrate that BMI and AC are positively associated with hemoglobin levels in patients with CKD. Hemoglobin levels in male patients significantly increased with increasing BMI and $\mathrm{AC}$, and were accompanied by higher CRP levels. This was not the case in female patients. Although hemoglobin levels increased with increased BMI and $\mathrm{AC}$ in female patients with BMI less than $25 \mathrm{~kg} / \mathrm{m}^{2}$ or small $\mathrm{AC}$, hemoglobin levels plateaued once BMI reached $25 \mathrm{~kg} / \mathrm{m}^{2}$ or the $\mathrm{AC}$ reached $80 \mathrm{~cm}$.

In an analysis of data from the third National Health and Nutrition Examination Survey (NHANES), conducted in the USA ( $n=14,846)$, BMI did not affect hemoglobin levels; however, the association not assessed according to sex [17]. Kohno et al. analyzed a Japanese cohort of 1029 patients and found that BMI was positively associated with biomarkers of erythropoiesis in a post hoc analysis by sex [32]. Vuong et al. demonstrated similar findings in 6766 NHANES patients; hemoglobin levels increased with increasing $\mathrm{AC}$ in both male and female patients [6]. Traissac et al. reported that, in a non-CKD cohort of 1689 female patients and 930 male patients, the prevalence of anemia (hemoglobin $<12.0 \mathrm{~g} / \mathrm{dL}$ ) was significantly higher in females with BMI $\geq 25 \mathrm{~kg} / \mathrm{m}^{2}$ than in males with BMI $\geq 25 \mathrm{~kg} / \mathrm{m}^{2}$ [33]. Thus, in general, BMI and AC appear to be positively associated with hemoglobin levels in the nonCKD population. However, these associations are likely to be attenuated in females with BMI higher than or equal to the threshold of $25 \mathrm{~kg} / \mathrm{m}^{2}$. Overall, the association between hemoglobin levels and BMI appears to be maintained in patients with CKD, even though the hemoglobin levels are in the anemic range.

The associations between body composition and hemoglobin levels were affected by CKD severity, and disappeared once CKD reached stage 5 . This may be due to inadequate EPO production and erythropoiesis in the uremic condition. EPO concentration was not associated with $\mathrm{BMI}$ or $\mathrm{AC}$ in the multivariate regression models adjusting for CKD stage; however, EPO levels increased with increasing $\mathrm{BMI}$ and $\mathrm{AC}$ in patients with stage $3 \mathrm{CKD}$. Thus, adiposity may be associated with increased EPO 
production in stage $3 \mathrm{CKD}$, and this effect may be diminished in stage 4 CKD and beyond.

In both sexes, hemoglobin levels were higher in patients with normal BMI than in those with low BMI. This might be linked to undernourishment. However, it is not clear why hemoglobin levels did not increase with increasing adiposity in female patients, as levels of EPO and iron metabolism biomarkers increased with increase in BMI and AC to a similar extent in both sexes. Sex hormones could play a role; testosterone has been reported to have a stimulatory effect on erythropoiesis [34]. Testosterone levels in male patients with high BMI between 25 and $30 \mathrm{~kg} / \mathrm{m}^{2}$ appear to be similar to those in male patients with normal BMI, while the levels in male patients with BMI $>30 \mathrm{~kg} / \mathrm{m}^{2}$ were decreased compared to those in male patients with normal BMI [35]. Thus, testosterone might stimulate erythropoiesis in male patients with BMI $\leq 30 \mathrm{~kg} / \mathrm{m}^{2}$; however, erythropoiesis might be associated with other unknown mechanisms in men with BMI $>30 \mathrm{~kg} / \mathrm{m}^{2}$. Increased CKD stage may influence testosterone levels; the prevalence of hypogonadism (testosterone $<10 \mathrm{nmol} / \mathrm{L}$ ) is $17 \%$ in stages $1-2 \mathrm{CKD}, 34 \%$ in stage $3 \mathrm{CKD}, 38 \%$ in stage 4 CKD, and $57 \%$ in stage 5 CKD [36]. Therefore, an effect of testosterone might be sustained during CKD stages 3 and 4.

Adiposity enhances low iron status and increases CRP and ferritin levels in the non-CKD population [15-17]; thus, adiposity-related inflammation can cause functional iron deficiency. However, we were not able to confirm this association in patients with CKD. Although CRP levels increased with increasing BMI and AC in stages 3-5 CKD, and ferritin levels increased according to BMI category, BMI and $\mathrm{AC}$ did not affect transferrin saturation levels in patients with CKD. Ausk et al. reported that ferritin levels in individuals in the general population with BMI between 25 and $30 \mathrm{~kg} / \mathrm{m}^{2}$ were higher than in those with BMI $>25 \mathrm{~kg} / \mathrm{m}^{2}$ [17]. Similarly, Cepeda-Lopez et al. reported that CRP levels and iron status were associated with BMI, with higher CRP levels in females with BMI between 25 and $30 \mathrm{~kg} / \mathrm{m}^{2}$ than in females with BMI between 18.5 and $24.5 \mathrm{~kg} / \mathrm{m}^{2}$. However, iron status in the $25-30 \mathrm{~kg} / \mathrm{m}^{2}$ BMI group resembled that of the $18.5-24.5 \mathrm{~kg} / \mathrm{m}^{2}$ BMI group, and iron deficiency rose in patients with a BMI $\geq 30 \mathrm{~kg} / \mathrm{m}^{2}$ [15]. Thus, iron deficiency may not have been evident in the present study due to the smaller numbers of participants with BMI $\geq 30 \mathrm{~kg} / \mathrm{m}^{2}$.

The present study has several limitations. The patient cohort was relatively small, and there was missing biomarker data, especially for iron metabolism. Several patients were excluded from the 1- and 2-year follow-up, as patients in advanced stages of CKD at baseline were referred for dialysis and did not visit our hospital after completing the baseline measurements. Thus, the patients who left the study may have affected our findings at later time periods. In addition, anemia was managed according to the Japanese guidelines for anemia in patients with CKD
[37], and strategies for anemia management, such as ESA therapy and ferrotherapy, were determined by the patient's primary doctors. Moreover, there was an uneven distribution of CKD stages in the BMI and AC groups in the present study. Finally, we cannot speak to the pathogenesis of increased erythropoiesis with increased adiposity, as we did not assess causal associations.

In conclusion, body composition appears to influence erythropoiesis; however, adiposity may be only associated with increased erythropoiesis in male patients. In contrast, body composition does not appear to hamper iron metabolism in patients with CKD who are not on dialysis. Further research is required, with a larger cohort, to confirm the impact of adiposity on erythropoiesis.

Acknowledgements This study was conducted by principal investigators at the following medical centers: Japan Community Health Care Organization Sendai Hospital (Miyagi), JA Toride Medical Center (Ibaraki), Jichi Medical University (Tochigi), Tokyo Women's Medical University Hospital (Tokyo), St. Luke's International Hospital (Tokyo), Showa University Hospital (Tokyo), Showa University Yokohama Northern Hospital (Kanagawa), Showa University Fujigaoka Hospital (Kanagawa), Gifu Prefectural General Medical Center (Gifu), Kasugai Municipal Hospital (Aichi), Tosei General Hospital (Aichi), Osaka University Hospital (Osaka), Osaka General Medical Center (Osaka), Osaka City General Hospital (Osaka), Kurashiki Central Hospital (Okayama), Fukuoka Red Cross Hospital (Fukuoka), and Iizuka Hospital (Fukuoka). This study was supported by research funds from Kyowa Hakko Kirin Co., Ltd, with no restrictions on publication.

\section{Compliance with ethical standards}

Conflict of interest T.A. has consulted for and received lecture fees from Kyowa Hakko Kirin. K.N. has consulted for and received a research support grant from Kyowa Hakko Kirin. H.H., K.O. and A.H. have no conflicts of interest to declare.

Ethical approval All procedures performed in studies involving human participants were in accordance with the ethical standards of the institutional and/or national research committee at the institutes where the studies were conducted and with the 1964 Helsinki declaration and its later amendments or comparable ethical standards.

Informed consent Informed consent was obtained from all individual participants included in the study.

Open Access This article is distributed under the terms of the Creative Commons Attribution 4.0 International License (http://creative commons.org/licenses/by/4.0/), which permits unrestricted use, distribution, and reproduction in any medium, provided you give appropriate credit to the original author(s) and the source, provide a link to the Creative Commons license, and indicate if changes were made.

\section{References}

1. Hsu CY, McCulloch CE, Curhan GC. Epidemiology of anemia associated with chronic renal insufficiency among adults in the United States: results from the Third National Health and Nutrition Examination Survey. J Am Soc Nephrol. 2002;13:504-10. 
2. Tsubakihara Y, Gejyo F, Nishi S, et al. High target hemoglobin with erythropoiesis-stimulating agents has advantages in the renal function of non-dialysis chronic kidney disease patients. Ther Apher Dial. 2012;16:529-40.

3. Iseki K, Kohagura K. Anemia as a risk factor for chronic kidney disease. Kidney Int Suppl. 2008;107:S4-9.

4. Babitt JL, Lin HY. Mechanisms of anemia in CKD. J Am Soc Nephrol. 2012;23:1631-4.

5. Chang JS, Chen YC, Owaga E, et al. Interactive effects of dietary fat/carbohydrate ratio and body mass index on iron deficiency anemia among Taiwanese women. Nutrients. 2014;6:3929-41.

6. Vuong J, Qiu Y, La M, et al. Reference intervals of complete blood count constituents are highly correlated to waist circumference: should obese patients have their own "normal values?". Am J Hematol. 2014;89:671-7.

7. Coimbra S, Catarino C, Santos-Silva A. The role of adipocytes in the modulation of iron metabolism in obesity. Obes Rev. 2013;14:771-9.

8. Barbieri M, Ragno E, Benvenuti E, et al. New aspects of the insulin resistance syndrome: impact on haematological parameters. Diabetologia. 2001;44:1232-7.

9. Sawada K, Krantz SB, Dessypris EN, et al. Human colonyforming units-erythroid do not require accessory cells, but do require direct interaction with insulin-like growth factor I and/or insulin for erythroid development. J Clin Invest. 1989;83:1701-9.

10. Ratajczak J, Zhang Q, Pertusini E, et al. The role of insulin (INS) and insulin-like growth factor-I (IGF-I) in regulating human erythropoiesis. Studies in vitro under serum-free conditionscomparison to other cytokines and growth factors. Leukemia. $1998 ; 12: 371-81$.

11. Miyagawa S, Kobayashi M, Konishi N, et al. Insulin and insulinlike growth factor I support the proliferation of erythroid progenitor cells in bone marrow through the sharing of receptors. $\mathrm{Br}$ J Haematol. 2000;109:555-62.

12. Tsuda K, Nishio I. Leptin and membrane fluidity of erythrocytes in essential hypertension. An electron paramagnetic resonance investigation. Am J Hypertens. 2004;17:375-9.

13. Tsuda K. Adiponectin and membrane fluidity of erythrocytes in normotensive and hypertensive men. Obesity (Silver Spring). 2006;14:1505-10.

14. Barazzoni R, Gortan Cappellari G, et al. The association between hematological parameters and insulin resistance is modified by body mass index-results from the North-East Italy MoMa population study. PLoS ONE. 2014;9:e101590.

15. Cepeda-Lopez AC, Osendarp SJ, Melse-Boonstra A, et al. Sharply higher rates of iron deficiency in obese Mexican women and children are predicted by obesity-related inflammation rather than by differences in dietary iron intake. Am J Clin Nutr. 2011;93:975-83.

16. Gartner A, Berger J, Bour A, et al. Assessment of iron deficiency in the context of the obesity epidemic: importance of correcting serum ferritin concentrations for inflammation. Am J Clin Nutr. 2013;98:821-6.

17. Ausk KJ, Ioannou GN. Is obesity associated with anemia of chronic disease? A population-based study. Obesity (Silver Spring). 2008;16:2356-61.

18. Neymotin F, Sen U. Iron and obesity in females in the United States. Obesity (Silver Spring). 2011;19:191-9.

19. Ganz T, Nemeth E. Iron balance and the role of hepcidin in chronic kidney disease. Semin Nephrol. 2016;36:87-93.

20. Fraenkel PG. Understanding anemia of chronic disease. Hematology Am Soc Hematol Educ Program 2015; 2015:14-8.
21. Spoto B, Pisano A, Zoccali C. Insulin resistance in chronic kidney disease: a systematic review. Am J Physiol Renal Physiol. 2016;311:F1087-108.

22. Lim CC, Teo BW, Tai ES, et al. Elevated serum leptin, adiponectin and leptin to adiponectin ratio is associated with chronic kidney disease in Asian adults. PLoS ONE. 2015;10:e122009.

23. Alix PM, Guebre-Egziabher F, Soulage CO. Leptin as an uremic toxin: deleterious role of leptin in chronic kidney disease. Biochimie. 2014;105:12-21.

24. Mercadal L, Metzger M, Haymann JP, et al. The relation of hepcidin to iron disorders, inflammation and hemoglobin in chronic kidney disease. PLoS ONE. 2014;9:e99781.

25. Uehata T, Tomosugi N, Shoji T, et al. Serum hepcidin-25 levels and anemia in non-dialysis chronic kidney disease patients: a cross-sectional study. Nephrol Dial Transplant. 2012;27:1076-83

26. Imai E, Matsuo S, Makino $\mathrm{H}$, et al. Chronic Kidney Disease Japan Cohort (CKD-JAC) study: design and methods. Hypertens Res. 2008;31:1101-7.

27. Imai E, Matsuo S, Makino H, et al. Chronic Kidney Disease Japan Cohort study: baseline characteristics and factors associated with causative diseases and renal function. Clin Exp Nephrol. 2010;14:558-70.

28. Inaguma $\mathrm{D}$, Imai $\mathrm{E}$, Takeuchi $\mathrm{A}$, et al. Risk factors for CKD progression in Japanese patients: findings from the Chronic Kidney Disease Japan Cohort (CKD-JAC) study. Clin Exp Nephrol. 2017;21:446-56.

29. Tanaka K, Watanabe T, Takeuchi A, et al. Cardiovascular events and death in Japanese patients with chronic kidney disease. Kidney Int. 2017;91:227-34.

30. WHO Expert Consultation. Appropriate body-mass index for Asian populations and its implications for policy and intervention strategies. Lancet. 2004;363:157-63.

31. Alberti KG, Eckel RH, Grundy SM, et al. Harmonizing the metabolic syndrome: a joint interim statement of the International Diabetes Federation Task Force on Epidemiology and Prevention; National Heart, Lung, and Blood Institute; American Heart Association; World Heart Federation; International Atherosclerosis Society; and International Association for the Study of Obesity. Circulation. 2009;120:1640-5.

32. Kohno K, Narimatsu H, Shiono Y, et al. High serum adiponectin level is a risk factor for anemia in Japanese men: a prospective observational study of 1,029 Japanese subjects. PLoS ONE. 2016;11:e0165511.

33. Traissac P, El Ati J, Gartner A, et al. Gender inequalities in excess adiposity and anaemia combine in a large double burden of malnutrition gap detrimental to women in an urban area in North Africa. Public Health Nutr. 2016;19:1428-37.

34. Shahidi NT. Androgens and erythropoiesis. N Engl J Med. 1973;289:72-80.

35. Dhindsa S, Miller MG, McWhirter CL, et al. Testosterone concentration in diabetic and nondiabetic obese men. Diabetes Care. 2010;33:1186-92.

36. Yilmaz MI, Sonmez A, Qureshi AR, et al. Endogenous testosterone, endothelial dysfunction, and cardiovascular events in men with nondialysis chronic kidney disease. Clin J Am Soc Nephrol. 2011;6:1617-25.

37. Tsubakihara Y, Nishi S, Akiba T, et al. 2008 Japanese Society for Dialysis Therapy: guidelines for renal anemia in chronic kidney disease. Ther Apher Dial. 2010;14:240-75. 